\title{
Effect of Nonsurgical Treatment on Salivary HGF Levels in Population with Periodontal Disease: A Quasi-experimental Study
}

\author{
Dalip Alreja ${ }^{1}$, Jyoti R Rao ${ }^{2}$, Sakshi Kataria ${ }^{3}$, Dhaval A Faterpenkar ${ }^{4}$
}

\begin{abstract}
Aim: To assess the effect of nonsurgical treatment on salivary hepatocyte growth factor (sHGF) levels in a population with periodontal disease: a quasi-experimental study.

Methods: Eighty-one patients (aged 30-70 years) were divided into three groups based on the gingival index, probing depth, clinical attachment loss, and radiographic evidence of bone loss: healthy (group I), gingivitis (group II), and chronic periodontitis (group III). Saliva samples were collected from these groups at baseline. At 8 weeks, saliva samples were collected again from group II and group III after the patients went through nonsurgical periodontal treatment. The levels of HGF were estimated using enzyme-linked immunosorbent assay (ELISA). The clinical parameters and HGF levels among all groups were analyzed using a one-way analysis of variance (ANOVA) using SPSS 17 version.

Results: At baseline, the highest mean HGF concentration in saliva was observed for group III ( $3455.83 \pm 1463.44 \mathrm{pg} / \mathrm{mL})$, and the least in group I $(469.43 \pm 317.13 \mathrm{pg} / \mathrm{mL})$. Following nonsurgical periodontal treatment, the mean HGF concentration decreased significantly in group III and group II ( $p<0.05)$. A significant positive correlation between clinical parameters and HGF levels was also seen $(p<0.05)$.

Conclusion: HGF concentration showed a positive correlation with the progression of periodontal disease.

Clinical significance: Following nonsurgical periodontal therapy, the levels of HGF decreased significantly, suggesting that HGF could be useful for monitoring the response to periodontal therapy.

Keywords: Biomarker, Boster's human HGF ELISA Kit ${ }^{\oplus}$, Chronic periodontitis, Enzyme-linked immunosorbent assay, Gingivitis, Hepatocyte growth factor, Periodontal disease, Salivary hepatocyte growth factor.

Euroasian Journal of Hepato-Gastroenterology (2020): 10.5005/jp-journals-10018-1320
\end{abstract}

\section{INTRODUCTION}

Periodontal disease is an inflammatory disorder with multifactorial etiology, characterized clinically by gingival inflammation, pathological deepening of gingival sulcus, and loss of clinical attachment and radiographically by the loss of supporting alveolar bone. The main factor responsible for the progression of periodontal disease is the deepening of the periodontal pocket resulting in apical migration of rete pegs, with a subsequent loss of collagen fibers, migration of junctional epithelial cells along the root surface wall, and invasion of this space by connective tissue. ${ }^{1,2}$ The protein concentration of gingival crevicular fluid (GCF) can be assessed here by the breakdown of the connective tissue. ${ }^{3}$ There are several mediators or markers of the periodontal disease activity present in the GCF, and these markers complement component cytokines. Hepatocyte growth factor (HGF) is one such cytokine. It plays an important role in facilitating epithelial migration as well as vascular epithelial growth during pocket formation. ${ }^{4}$

The mesenchymal cells secrete primarily a heterodimeric protein, that is, scatter factor (SF) most popularly known as HGF. It functions in a paracrine manner on the surrounding epithelial/endothelial cells. ${ }^{5,6} \mathrm{HGF}$ virtually affects every tissue in the body starting from the nervous system to the immune and reticuloendothelial systems, apart from affecting the liver. It impedes cell growth, instigates morphogenesis, stimulates T-cell adhesion to endothelium and migration, supplements neuron survival, and controls erythroid differentiation. It is recognized to be a potent angiogenic factor and \begin{tabular}{l}
\hline \hline 1,2Department of Periodontics, Goa Dental College and Hospital, \\
Panjim, Goa, India \\
${ }^{3}$ Department of Public Health Dentistry, Sudha Rustagi College of \\
Dental Sciences and Research, Faridabad, Haryana, India \\
${ }^{4}$ Department of Microbiology, Goa Medical College, Panaji, Goa, India \\
Corresponding Author: Sakshi Kataria, Department of Public \\
Health Dentistry, Sudha Rustagi College of Dental Sciences and \\
Research, Faridabad, Haryana, India, Phone: +91 9810132292, e-mail: \\
sakshikataria87@gmail.com \\
How to cite this article: Alreja D, Rao JR, Kataria S, et al. Effect of \\
Nonsurgical Treatment on Salivary HGF Levels in Population with \\
Periodontal Disease: A Quasi-experimental Study. Euroasian J Hepato- \\
Gastroenterol 2020;10(2):51-55. \\
Source of support: Nil \\
Conflict of interest: None \\
\hline \hline
\end{tabular}

morphogen. HGF also induces epithelial cell mitogenesis, induces cell motility, and promotes matrix invasion. ${ }^{8}$

Inflammatory cytokines, such as interleukin (IL)-1 $\alpha$, IL-1 $\beta$, tumor necrosis factor- $\alpha$ (TNF- $\alpha$ ), and prostaglandin E2 (PGE2) enhances the production of HGF by gingival and periodontal fibroblast ligament. ${ }^{9,10}$ Additionally, Gram-positive bacteria, such as Prevotella intermedia (periodontopathogenic bacteria) ${ }^{11}$ : lipoteichoic acid and a glycoprotein portion and Porphyromonas gingivalis ${ }^{12}$ : fimbriae; cell 
surface components which enhance HGF production in gingival fibroblasts in culture.

The HGF concentration in the GCF is found to be significantly higher at periodontally compromised sites in patients with periodontitis as compared to that in healthy patients. A study by Nagaraja and Pradeep has shown that nonsurgical periodontal treatment decreases the GCF HGF levels in patients with chronic periodontitis. ${ }^{13-15}$ Although several studies have found an association between periodontal disease and HGF levels in GCF and saliva, knowledge of the effect of treatment of periodontal disease on GCF and salivary HGF levels is scarce.

The dental literature to date has documented limited studies that have been conducted to assess the effect of nonsurgical treatment on salivary HGF levels in a population with periodontal disease: a quasi-experimental study. ${ }^{16-22}$ Hence in light of the aforementioned facts, this quasi-experimental study was designed to estimate and compare the levels of HGF in the saliva of patients with clinically healthy periodontium, gingivitis, and chronic periodontitis after nonsurgical periodontal treatment.

\section{Materials and Methods}

\section{Study Population/Criteria for Selection}

Using G Power software (version 3.0.10), the sample size was calculated with an effect size of $0.24,5 \%$ level of precision, $95 \%$ confidence level, and $80 \%$ power of the study. The minimum sample size for the study was 72 .

The present study was carried out over a period of 9 months (March-November, 2014). Eighty-one patients (41 males and 40 females) with a mean age of $36.40 \pm 4.29$ years attending the outpatient section of the Department of Periodontics, Goa Dental College and Hospital, Goa, India, were recruited for the study.

\section{Inclusion criteria}

- Patients within the age range 30 to 70 years,

- Patients having no history of periodontal treatment in the previous 6 months,

- Patients having no history with respect to medications-antiinflammatory, antibiotics, or immunosuppressive drugs in the last 6 months.

\section{Exclusion criteria}

- Pregnant or lactating females,

- Patients with a history of diabetes, hypertension, liver disorders, bleeding disorders, antiepileptic therapy, salivary gland disorders, oral mucosal lesions, and any condition necessitating antibiotic premedication for dental appointments,

- Patients with fewer than 22 teeth, active carious lesions, presence of aggressive periodontitis, periodontal abscess, or necrotizing ulcerative gingivitis or periodontitis,

- Smokers and alcoholics patients.

The GDCH institutional ethical committee, Goa Dental College and Hospital, Goa University, Goa, India, provided approval for the study. Written informed consent was obtained from those who agreed to participate voluntarily in this study after reading the information sheet.

\section{Clinical Measurements and Subject Grouping}

To determine the clinical periodontal status, periapical radiographs using the long-cone technique was performed for full-mouth periodontal probing and charting. Radiographic bone loss was recorded and dichotomized (presence or absence) to differentiate chronic periodontitis patients from other groups. One clinical examiner performed all the clinical measurements, who was trained and calibrated in the department under the supervision of an examiner with 12 years of clinical expertise. The intraexaminer agreement was 0.85 . The patients of the pilot study were not included in the main research.

Gingival index $(\mathrm{GI})^{23}$, probing depth (PD), clinical attachment loss (CAL), and radiographic evidence of bone loss were recorded. Patients were categorized into three groups.

- Group I (healthy) N=27 patients, with clinically healthy periodontium and with no evidence of disease $(\mathrm{Gl}<1$; $\mathrm{PD} \leq 3 \mathrm{~mm} ; \mathrm{CAL}=0 \mathrm{~mm}$; and radiographic absence of crestal bone loss).

- Group Il (gingivitis) $\mathrm{N}=27$ patients, with clinical signs of gingival inflammation and no evidence of attachment loss $(G I \geq 1$; $\mathrm{PD} \leq 3 \mathrm{~mm} ; \mathrm{CAL}=0 \mathrm{~mm}$; and radiographic absence of crestal bone loss).

- Group III (chronic periodontitis) N=27 patients, with clinical signs of gingival inflammation and attachment loss $(\mathrm{Gl} \geq 1 ; \mathrm{PD} \geq 5 \mathrm{~mm}$; $C A L \geq 1 \mathrm{~mm}$; and radiographic evidence of crestal bone loss).

Periodontal treatment (SRP) was performed for group II (gingivitis) and group III (chronic periodontitis) patients at the same appointment after saliva collection.

After 8 weeks, second saliva samples were collected from the patients in group II $\mathrm{N}=25$; and group III; $\mathrm{N}=25$. During their second visit, plaque control measures were performed and their plaque index (PI) was monitored to supervise their personal oral hygiene maintenance.

\section{Saliva Sampling}

To avoid probing-associated contamination of saliva with blood at the inflamed sites, all clinicoradiological examinations were performed one day prior, before the saliva was collected. Patients were instructed not to eat or drink 3 hours before sample collection on the following day of that visit.

In the morning, on arrival, patients were asked to rinse their mouth with $15 \mathrm{~mL}$ of water (to wash out exfoliated cells) and immediately, unstimulated passive drool of whole saliva sample 0.5 to $1 \mathrm{ml}$ was collected, in a sterile disposable plastic container.

Samples were preserved in ice for 2 hours and then transferred to $1.5 \mathrm{ml}$ microtubes. At $4{ }^{\circ} \mathrm{C}$ speed of $3800 \mathrm{rpm}$, centrifugation was done for $10 \mathrm{~min}$ to remove particulate matter and bacteria. The supernatant (middle third) was stored at $-40^{\circ} \mathrm{C}$ till the time of assay. ${ }^{23}$

\section{HGF Determination}

- Boster's human HGF ELISA Kit ${ }^{\circledR}$ was used to evaluate the concentration of HGF in saliva. The standard sandwich ELISA was performed at the Department of Microbiology, Goa Medical College and Hospital, Goa, India.

- A 96-well microtiter plate precoated with monoclonal antibody from mouse specific for HGF was used.

- Human HGF standards ( $0.1 \mathrm{~mL}$ per well of $8000,4000,2000,1000$, $500,250,125 \mathrm{pg} / \mathrm{mL}$ ) and $0.1 \mathrm{~mL}$ saliva samples were pipetted into the wells and incubated at $37^{\circ} \mathrm{C}$ for 90 minutes.

- HGF, if present in the sample, was bound by the immobilized monoclonal antibody.

- After washing away any unbound substances, $0.1 \mathrm{ml}$ of biotinylated detection polyclonal antibody from goat specific for HGF was added to the wells and incubated at $37^{\circ} \mathrm{C}$ for 60 minutes. 
- The plate was washed three times with $0.01 \mathrm{M}$ phosphate buffer to remove any unbound antibodies. Avidin-biotin-peroxidase complex $(A B C)$ working solution is added, incubated at $37^{\circ} \mathrm{C}$ for 30 minutes and washed five times with $0.01 \mathrm{M}$ phosphate buffer.

- Substrate solution $(0.09 \mathrm{~mL})$ was added to the wells and incubated at $37^{\circ} \mathrm{C}$ in dark for $20-25$ minutes.

- Color developed in proportion to the amount of HGF bound in the initial step. The color development was stopped using a stop solution $(0.1 \mathrm{~mL})$, and the intensity of the color was measured spectrophotometrically using a microplate reader set at $450 \mathrm{~nm}$ within 30 minutes.

- The concentration of HGF in the tested samples was estimated using the standard curve plotted using the optical density values with the standards.

- All samples and standards were assayed in duplicate as suggested by the manufacturer.

\section{Statistical Analysis}

Statistical analysis of the data was performed using a software program SPSS version 17.0 (SPSS Inc., Chicago, IL, USA). Mean and standard deviations for age, PI, GI, PD, CAL, and saliva HGF levels of the participants were analyzed. The normality assumption of the data was tested using the Shapiro-Wilk W-test. Analysis of variance was performed for more than three independent groups. Post hoc testing (Bonferroni comparisons test) was performed to explore the differences between any two groups. Pearson's correlation analysis was used to test any correlation between the HGF concentration in saliva with PI, GI, PD, and CAL. Statistical significance was established at $p<0.05$.

\section{Results}

\section{Characteristics of the Study Population}

All samples in each group tested positive for HGF assay. The distribution of patients and their mean concentration and the range of HGF levels in all groups, along with the standard deviations, are shown in Tables 1 and 2, respectively.

The saliva HGF levels were the highest in patients with chronic periodontitis followed by gingivitis patients and patients with healthy periodontium. By using ANOVA, a significant difference in mean values for HGF concentration was observed between the

Table 1: Distribution of patients among the three groups at baseline and follow-up visits

\begin{tabular}{lll}
\hline & Baseline & At follow-up visits \\
\hline Group I & $\mathrm{N}=27$ & - \\
Group II & $\mathrm{N}=27$ & $\mathrm{~N}=25$ \\
Group III & $\mathrm{N}=27$ & $\mathrm{~N}=25$ \\
\hline
\end{tabular}

groups ( $p<0.05$ ). On pairwise comparisons using the Bonferroni test, statistically significant difference was observed among all pairs of groups $(p<0.05)$. Nonsurgical periodontal treatment led to a statistically significant proportional reduction in saliva levels of HGF in chronic periodontitis and gingivitis patients (Fig. 1).

Comparison of the PI, GI, PD, CAL, and sHGF scores showed a statistically significant difference among all the study groups. The HGF concentration increases with the progression of periodontal disease and the same decreases after treatment that is aimed at resolution of periodontal inflammation and gain in clinical attachment level (Table 3).

Pearson's correlation analysis was used to find correlation between the HGF concentration in saliva with clinical parameters$\mathrm{PI}, \mathrm{GI}, \mathrm{PD}$, and CAL. A statistically significant "positive moderate correlation" was found for all these clinical parameters, for all the groups $(p<0.001)$.

\section{Discussion}

Human periodontal diseases are inflammatory disorders that give rise to tissue destruction, as a result of complex interactions between pathogenic bacteria and the host's immune response. The hallmark of periodontitis is the loss of connective tissue attachment to the tooth. Several cytokines have been reported to act as chemoattractants for migrating epithelial cells. ${ }^{19,20}$ The search for diagnostic tests of periodontal disease activity is of paramount importance in current periodontal research. These include by-products of the host bacterial interactions like aspartate aminotransferase, $\beta$-glucuronidase, cathepsin-G, etc. HGF is one such factor that acts as a motogen, which is released in response to inflammation during periodontal destruction. ${ }^{21}$ Recently, the existence of HGF in GCF in both healthy

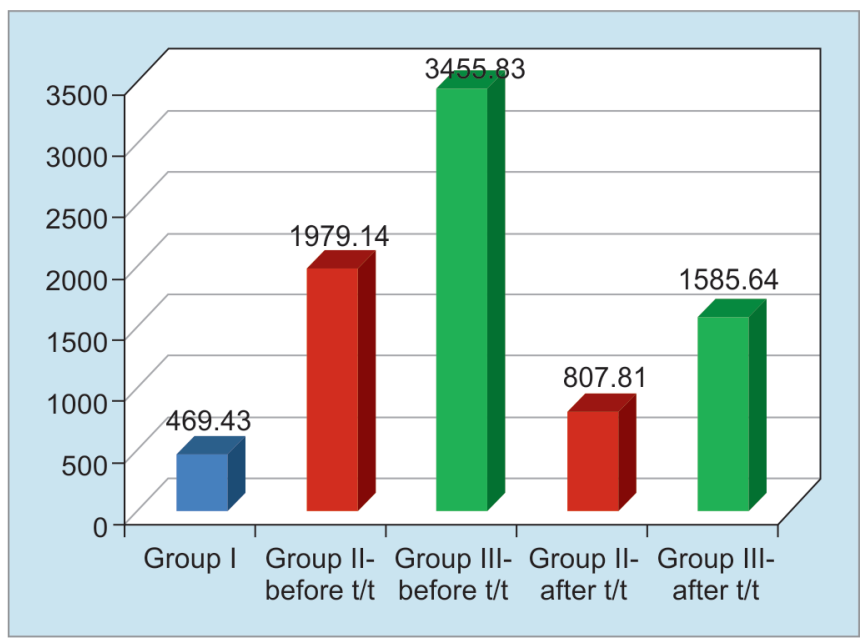

Fig. 1: Comparison of values of HGF in saliva $(\mathrm{pg} / \mathrm{mL})$

Table 2: Description of values of HGF in saliva $(\mathrm{pg} / \mathrm{mL})$

\begin{tabular}{lllll}
\hline & & \multicolumn{2}{c}{ Before treatment } & \multicolumn{2}{c}{ After treatment } \\
\hline Group & Mean \pm SD & Range & Mean \pm SD & Range \\
Group I: (healthy, $\mathrm{N}=27$ ) & $469.43 \pm 317.13$ & $88.47-1063.18$ & - & - \\
Group II: (gingivitis, $\mathrm{N}=27$ ) & $1979.14 \pm 1260.63$ & $147.44-4264.56$ & $807.81 \pm 375.95$ & $148.88-1401.34$ \\
Group III: (chronic periodontitis) & $3455.83 \pm 1463.44$ & $567.29-5640.98$ & $1585.64 \pm 565.44$ & $401.73-2817.35$ \\
\hline
\end{tabular}


Salivary HGF Levels in Population with Periodontal Disease

Table 3: Comparison of mean \pm SD different clinical parameters and HGF levels of saliva among groups at baseline and follow-up visits

\begin{tabular}{llllll}
\hline Parameters & Group I & Group II - before $t / t$ & Group III - before /t $t$ & Group II - after $t / t$ & Group III - after $t / t$ \\
\hline Plaque index (PI) & $0.45 \pm 0.96$ & $2.02 \pm 0.87$ & $2.46 \pm 0.94$ & $0.71 \pm 0.17$ & $0.73 \pm 0.21$ \\
Gingival index (GI) & $0.67 \pm 0.32$ & $1.77 \pm 0.41$ & $2.06 \pm 0.35$ & $0.77 \pm 0.19$ & $1.01 \pm 0.23$ \\
Probing depth (PD) & $2.04 \pm 0.23$ & $2.74 \pm 0.32$ & $5.62 \pm 0.43$ & $2.41 \pm 0.26$ & $3.46 \pm 0.43$ \\
Clinical attachment loss (CAL) & - & - & $5.97 \pm 0.27$ & - & $3.83 \pm 0.30$ \\
HGF in saliva (pg/mL) & $469.43 \pm 317.13$ & $1979.14 \pm 1260.63$ & $3455.83 \pm 1463.44$ & $807.81 \pm 375.95$ & $1585.64 \pm 565.44$ \\
\hline
\end{tabular}

ONE WAY ANOVA TEST FOR: PI, GI, PD, sHGF

Independent $t$ test: FOR CAL

LEVEL OF SIGNIFICANCE SET AT $p<0.05$

and diseased gingiva was demonstrated and was found to be positively correlated to IL-1 $\beta$ concentrations and clinical parameters of disease progression. ${ }^{15,22}$ HGF levels in GCF could be used as a sensitive marker for periodontal disease status. However, in a study by Kakimoto et al. no attempts were made to estimate HGF concentrations after periodontal therapy. ${ }^{17}$

Periodontal diagnosis using saliva has been the subject of considerable research activity. Proteins of host origin (i.e., enzymes, immunoglobulins), phenotypic markers (epithelial keratins), host cells, hormones (cortisol), bacteria and bacterial products, volatile compounds, and ions are being studied as proposed markers for monitoring periodontal disease. ${ }^{23}$ It has been postulated that analysis of the whole saliva holds greater promise than gland-specific saliva when considering the development of a diagnostic test for periodontal disease. ${ }^{24}$ Salivary levels of HGF have been positively correlated with clinical parameters like bleeding on probing and PD, and it has been suggested that salivary HGF may be a novel marker for periodontal diagnosis.

Hence, the present interventional study was undertaken to clinically and biochemically evaluate and compare the levels of HGF in saliva collected from patients with healthy periodontium, gingivitis, and chronic periodontitis, and after nonsurgical periodontal treatment in patients with gingivitis and chronic periodontitis. To achieve this, HGF levels in saliva from different groups were quantified and compared and probable correlation was studied between HGF levels and clinical parameters like $\mathrm{PI}$, $\mathrm{GI}, \mathrm{PD}$, and CAL.

HGF concentrations in saliva in our study were significantly lower than those of Magdalena et al., who reported a higher concentration of HGF in saliva in healthy and periodontitis groups. ${ }^{25}$ This may be related to the different sampling methods and differences in the racial backgrounds of the study patients. In our study, the mean HGF concentration $(\mathrm{pg} / \mathrm{mL})$ in the whole saliva exponentially increased in group II and group III as compared to group I (Fig. 1). The values of mean HGF concentration were substantially reduced in patients treated with scaling and root planning. Further, the treatment aimed at arresting periodontal disease progression resulted in a statistically significant reduction in the levels of HGF in saliva proportionally, which confirmed HGF's active role in periodontal attachment loss. Site-specific information on changes in HGF levels as a result of periodontal disease and after treatment was provided by our study.

The results of our study are similar to the interventional study conducted by Nagaraja and Pradeep. ${ }^{13}$ Authors conducted the study to assess HGF's concentration in GCF and to determine its association with periodontal disease progression. They found that HGF concentration increased proportionally with the progression of periodontal disease and showed a positive correlation with the clinical parameters. The significant decrease in levels of HGF ( $p<0.001$ ) following periodontal therapy suggested that HGF could be useful for monitoring the response to periodontal therapy. However, in contrast to the results of our study, Ohshima et al. did not find a statistically significant decrease in HGF concentration in GCF following SRP. ${ }^{16}$

Plaque index was recorded to assess oral hygiene. ${ }^{24}$ In our study, a statistically highly significant and positive correlation was found between $\mathrm{PI}$ and saliva HGF which suggests that an increase in $\mathrm{PI}$ leads to an increase in saliva $\operatorname{HGF}(\rho=+0.390, p<0.001)$. Plaque index was the highest in group III, followed by group II, group V, group IV, and group I. This finding positively supports the role of plaque in gingivitis and periodontitis and its correlation with the HGF level in saliva.

In our study, the difference in the PI between the gingivitis group (group II) and chronic periodontitis group (group III) was found to be statistically nonsignificant $(p>0.05)$, while the difference in $\mathrm{GI}$ and PD between these two groups was statistically significant $(p<0.05)$. This could be attributed to different microbial species harboring the dental plaque and is in turn substantiated by the specific plaque hypothesis ${ }^{24}$ which states that only certain plaque is pathogenic, and its pathogenicity depends on the presence of or increase in specific microorganisms. Wilczyńska-Borawska M et al. found a statistically significant positive correlation between HGF level in saliva and PI $(\rho=0.590, p<0.001)$, which is in accordance with the present study. ${ }^{25}$

The degree of gingival inflammation was measured by GI. ${ }^{23}$ In the present study, statistically highly significant and a positive correlation was found between $\mathrm{Gl}$ and saliva HGF, which suggests that an increase in Gl leads to an increase in saliva HGF concentration. ( $\rho=+0.753, p<0.001)$. The gingival index was the highest in group III, followed by group II, group V, group IV, and group I. The findings of the present study are in accordance with the study conducted by Nagaraja et al. ${ }^{13}$

In the present study, statistically highly significant and a positive correlation was found between pocket PD and saliva HGF which suggests that an increase in pocket $P D$ leads to an increase in saliva HGF concentration $(\rho=+0.802, p<0.001)$. Wilczyńska-Borawska M found results that are not in agreement with the present study. ${ }^{23}$ The probable reason for variation in the results could be the difference in the mean PD of the patients included in the study. The mean PD of chronic periodontitis patients in our study was 5.6 (5.0-6.3) $\mathrm{mm}$ in comparison to the mean PD of $3.0(1.8-5.9) \mathrm{mm}$ in this study by Wilczyńska-Borawska M. ${ }^{25}$

The loss of clinical attachment is an important clinical event in chronic periodontitis. In the present study, a statistically highly significant and positive correlation was found between CAL and 
saliva HGF, which suggests that an increase in CAL leads to an increase in saliva HGF concentration. $(\rho=+0.626, p<0.001)$. Clinical attachment loss was higher in group III as compared to group V ( $p<0.001)$. Group I, group II, and group IV did not have any CAL. The results of the present study are supported by Rudrakshi et al. ${ }^{14}$

Whole or mixed saliva is a complex fluid mixture derived from the major and minor salivary glands, and it contains contributions from the GCF, oral bacteria, cells, and other sources. Although saliva collection is easier than GCF collection, identification of the exact site of disease activity is difficult with saliva. This could have been one of the limitations of the present study. Secondly, the test could not be performed on patients with a history of systemic disease or any other acute infections since the above-mentioned conditions can affect the level of the analyte to be measured. ${ }^{26}$

\section{CONCLUSION}

The present research postulates that, with periodontal destruction, there is a substantial increase in the HGF concentration in saliva. However, longitudinal studies spanning over a longer time with a larger sample size may be beneficial in confirming the impact of HGF level fluctuations on periodontal disease extent and severity and to validate HGF as a "novel marker" of periodontal destruction and repair. A search for therapeutic drugs to prevent the formation or to modify the actions of HGF is a must. The development of a rapid chair-side diagnostic kit is the need of the hour.

\section{ACKnOWLedgment}

Nil

Manufacturer Name: Human HGF ELISA Kit PicoKine ${ }^{\mathrm{TM}}$ (Boster Biological Technology, Pleasanton CA, USA, Catalogue \# EK0369).

\section{References}

1. Ohshima M, Noguchi Y, Ito M, Maeno M, Otsuka K. Hepatocyte growth factor secreted by periodontal ligament and gingival fibroblasts is a major chemoattractant for gingival epithelial cells. J Periodontal Res 2001;36:377-383.

2. Ohshima M, Nishiyama T, Yamazaki Y, Yokosuka R, Maeno M, Otsuka K. Hepatocyte growth factor is a predominant chemoattractant for gingival epithelial cells produced by radicular cyst-derived fibroblast like cells. J Oral Sci 2000;42:101-106.

3. Attstrom R, Laural $A B$, Lashsson V. Complement factors in gingival crevice material from healthy and inflamed gingiva in humans. $J$ Periodontal Res 1974;10:19-27.

4. Gherardi E, Stoker M. Hepatocyte growth factor/scatter factor. Cancer Cells 1991;3:227.

5. Sonnenberg E, Meyer D, Weidner KM, Birchmeier W. Scatter factor/ hepatocyte growth factor and its receptor, the c-met tyrosine kinase, can mediate a signal exchange between mesenchyme and epithelia during mouse development. J Cell Biol 1993;123:223-235.

6. Weidner KM, Sachs M, Birchmeier W. The Met receptor tyrosine kinase transduces motility, proliferation, and morphogenic signals of scatter factor/hepatocyte growth factor in epithelial cells. J Cell Biol 1993;121: 145-154.

7. Zarnegar R, Michalopoulos GK. The many faces of hepatocyte growth factor: From hepatopoiesis to hematopoiesis. J Cell Biol 1995;129:1177-1180.
8. Montesano R, Matsumoto K, Nakamura T, Orci L. Identification of a fibroblast-derived epithelial morphogen as hepatocyte growth factor. Cell 1991;67:901-908.

9. Weidner KM, Behrens J, Vandekerckhove J, Birchmeier W. Scatter factor: Molecular characteristics and effects on the invasiveness of epithelial cells. J Cell Biol 1990; 111:2097-2108.

10. Tamura M, ArakakiN, TsubouchiH, TakadaH, Daikuhara Y. Enhancement of human hepatocyte growth factor production by IL-1 $\alpha$ and $-1 \beta$ and TNF- $\alpha$ by fibroblasts in culture. J Biol Chem 1993;268:8140-8145.

11. Ohnishi T, Suwa M, Oyama T, Arakaki N, Torii M, Daikuhara Y. Prostaglandin E2 predominantly induces production of hepatocyte growth factor/scatter factor in human dental pulp in acute inflammation. J Dent Res 2000;79:748-755.

12. Sugiyama A, Ogawa T, Daikuhara Y, Takada H. Enhancement of hepatocyte growth factor (scatter factor) production by human gingival fibroblasts in culture stimulated with Porphyromonas gingivalis fimbriae. J Med Microbiol 2000;49:319-325.

13. Nagaraja C, Pradeep AR. Hepatocyte growth factor levels in gingival crevicular fluid in health, disease, and after treatment. Journal of Periodontology. 2007 Apr;78(4):742-747.

14. Rudrakshi C, Srinivas N, Mehta DS. A comparative evaluation of hepatocyte growth factor levels in gingival crevicular fluid and saliva and its correlation with clinical parameters in patients with and without chronic periodontitis: A clinico-biochemical study. Journal of Indian Society of Periodontology. 2011 Apr;15(2):147.

15. Leo H. Gingival index, plaque index and retention index systems. J Periodontol 1967,38:610-616.

16. Ohshima M, Sakai A, Ito K, Otsuka K. Hepatocyte growth factor (HGF) in periodontal disease: Detection of HGF in gingival crevicular fluid. J Periodontal Res 2002;37:8-14.

17. Kakimoto K, Machigashira M, Ohnishi T, Kajihara T, Semba I, Setoguchi T, Tamura M, Izumi Y, Daikuhara Y. Hepatocyte growth factor in gingival crevicular fluid and the distribution of hepatocyte growth factor-activator in gingival tissue from adult periodontitis. Archives of oral biology. 2002 Sep 1;47(9):655-663.

18. Sato C, Tsuboi R, Shi CM, Rubin JS, Ogawa H. Comparative study of hepatocyte growth factor/scatter factor and keratinocyte growth factor effects on human keratinocytes. J Invest Dermatol 1995;104:958-963.

19. Mandel ID. Markers of periodontal disease susceptibility and activity derived from saliva. In: Johnson NW, editor. Risk markers for oral diseases. Periodontal diseases: Markers of disease susceptibility and activity. Cambridge: Cambridge University Press; vol.3, 1991. p. 228-253.

20. Kaufman E, Lamster IB. Analysis of saliva for periodontal diagnosis: A review. J Clin Periodontol 2000; 27:453-465.

21. Oshima M, Fugikawa K, Akutagawa HT, Ito K, Otsuka K. Hepatocyte growth factor in saliva: A possible marker for periodontal disease status. J Oral Sci 2002;44:35-39.

22. Silness $J$ and Loe H. Periodontal disease in pregnancy II. Correlation between oral hygiene and periodontal conditions. Acta Odont Scand 1964 ; 22: 112-134.

23. Wilczynska-Borawska M, Borawski J, Kovalchuk O, Chyczewski L, Stokowska W. Hepatocyte growth factor in saliva is a potential marker of symptomatic periodontal disease. Journal of Oral Science. 2006;48(2):47-50.

24. Loe $\mathrm{H}$, Silness J. Periodontal disease in pregnancy. I. Prevalence and severity. Acta Odontol Scand. 1963;21:533-551

25. Wilczyńska-Borawska M, Borawski J, Bagińska J, Małyszko J, Myśliwiec M. Hepatocyte growth factor in saliva of patients with renal failure and periodontal disease. Renal Failure. 2012 Sep 1;34(8):942-951.

26. Anil S, Vellappally S, Preethanath RS, Mokeem SA, AlMoharib HS, Patil $S$, Chalisserry EP, AI Kheraif AA. Hepatocyte growth factor levels in the saliva and gingival crevicular fluid in smokers with periodontitis. Disease Markers. 2014;2014. 